\title{
THE LOCAL RING OF THE GENUS THREE MODULUS SPACE AT KLEIN'S 168 SURFACE
}

\author{
BY H. E. RAUCH ${ }^{1}$ \\ Communicated by E. Calabi, November 28, 1966
}

1. Introduction. In [6], as a synthesis of earlier papers of mine, I give, in the form of a set of prescriptions for local coordinates, a description of $M^{\theta}$, the space of conformal equivalence classes of compact Riemann surfaces of genus $g$, as a complex space. Particular interest attaches to those points (surface classes) of $M^{\theta}$ representing surfaces admitting conformal self-maps (automorphisms) because, outside of certain cases for $g=1,2,3$ (over and above the elliptic and hyperelliptic involutions for $g=1,2$ ), these points are singular (nonuniformizable) points in the structure. In particular for $g \geqq 2$, where one needs $3 g-3$ complex parameters to describe $M^{\theta}$ near a generic point, one needs $3 g-3+\rho, \rho>0$, near one of the points in question. ${ }^{2}$ According to Prescription III ([6, p. 17]) the problem reduces to finding an irreducible basis for the homogeneous nonconstant, polynomial invariants of a finite group of linear transformations in $3 g-3$ variables, namely, the hermitian adjoint of the group induced on the quadratic differentials of a representative surface of the point in question by the conformal automorphism group of that surface.

For a finite nonabelian linear group, while there is an algorithm for computing some basis for the invariants (cf. Prescription III), there is notoriously no known algorithm for computing an irreducible basis, i.e., for discarding the superfluous ones. Accordingly I felt it of interest to illustrate the whole phenomenon by a nontrivial example. To anyone who has worked on the subject the one that immediately comes to mind is Klein's surface of genus three admitting as automorphism group a representation of the simple group of order 168 ([1], [3]). This example commends itself in that it is of "maximum complexity" in the sense that it admits its full quota according to Hurwitz [2] of $84(g-1)=168,(g=3)$ automorphisms (on the subject of such surfaces see the interesting papers [4], [5]). It develops, vide infra, that eleven invariants, i.e., $11=3 \cdot 3-3+5=6+5$, so $\rho=5$, are needed to generate the local ring of $M^{3}$ at Klein's surface class. ${ }^{2}$

${ }^{1}$ Research partially sponsored by the Air Force Office of Scientific Research, Office of Aerospace Research, U. S. Air Force, under AFOSR Grant No. AF-AFOSR1077-66.

${ }^{2}$ With $\rho$ relations on "syzygies," of course. 
2. The quadratic differentials on Klein's surface. Klein's surface $S$ is the compact Riemann surface of genus three obtained by identifying the upper half-plane under the principal congruence subgroup of level seven of the inhomogeneous modular group with appropriate conventions at cusps and vertices. The factor group $G$ acts on $S$ as its group of conformal automorphisms representing faithfully thereby the unique simple group of order 168.

Lemma 1 ([3, p. 444]). There is a basis $z_{1}, z_{2}, z_{3}$ of the abelian differentials of first kind on $S$ on which $G$ induces a representation $R_{3}(G)$ generated by

$$
\begin{aligned}
T: z_{1}^{\prime} & =\epsilon z_{1}, \quad z_{2}^{\prime}=\epsilon^{4} z_{2}, \quad z_{3}^{\prime}=\epsilon^{2} z_{3} \\
U: z_{1}^{\prime} & =a z_{1}+b z_{2}+c z_{3} \\
z_{2}^{\prime} & =b z_{1}+c z_{2}+a z_{3} \\
z_{3}^{\prime} & =c z_{1}+a z_{2}+b z_{3},
\end{aligned}
$$

where $\epsilon=e^{2 \pi i / 7}, \sqrt{ }-7=\epsilon+\epsilon^{2}+\epsilon^{4}-\epsilon^{3}-\epsilon^{5}-\epsilon^{6}$, and $a=\left(\epsilon^{5}-\epsilon^{2}\right) / \sqrt{ }-7$, $b=\left(\epsilon^{3}-\epsilon^{4}\right) / \sqrt{ }-7, c=\left(\epsilon^{6}-\epsilon\right) / \sqrt{ }-7$ are all real. $T$ has order 7 , and $U$ has order 2 .

LEMma 2. As a basis for the quadratic differentials on $S$ one can choose

$$
\begin{gathered}
\xi_{1}=z_{1}^{2}, \quad \xi_{2}=z_{2}^{2}, \quad \xi_{3}=z_{2}^{2}, \\
\xi_{4}=\sqrt{ } 2 z_{1} z_{2}, \quad \xi_{5}=\sqrt{ } 2 z_{2} z_{3}, \quad \xi_{6}=\sqrt{ } 2 z_{1} z_{8} .
\end{gathered}
$$

The representation $R_{6}(G)$ induced on $(2)$ by $R_{3}(G)$ is unitary.

Proof. $S$ is not hyperelliptic ([3, p. 437]), hence Noether's theorem ([6, Lemma 5]) implies that any six distinct quadratic products of the $z$ 's, in particular (2), span the quadratic differentials. By Lemma $1, R_{6}(G)$ is generated by

$$
\begin{aligned}
& T_{2}: \xi_{1}^{\prime}=\epsilon^{2} \xi_{1}, \xi_{2}^{\prime}=\epsilon \xi_{2}, \xi_{3}^{\prime}=\epsilon^{4} \xi_{3}, \xi_{4}^{\prime}=\epsilon^{5} \xi_{4}, \xi_{5}^{\prime}=\epsilon^{6} \xi_{5}, \xi_{6}^{\prime}=\epsilon^{3} \xi_{6}, \\
& U_{2}: \xi_{1}^{\prime}= a^{2} \xi_{1}+b^{2} \xi_{2}+c^{2} \xi_{3}+\sqrt{ } 2 a b \xi_{4}+\sqrt{ } 2 b c \xi_{5}+\sqrt{ } 2 a c \xi_{6}, \cdots, \\
& \xi_{4}^{\prime}= \sqrt{ } 2 a b \xi_{1}+\sqrt{ } 2 b c \xi_{2}+\sqrt{ } 2 a c \xi_{3}+\left(a c+b^{2}\right) \xi_{4} \\
&+\left(a b+c^{2}\right) \xi_{5}+\left(b c+a^{2}\right) \xi_{6}, \cdots,
\end{aligned}
$$

where the dots signify cyclic permutation of $a, b, c . T_{2}$ is clearly unitary. $U_{2}$ is real symmetric by inspection and of order 2 by Lemma 1 , hence real orthogonal, a fortiori unitary.

\section{Lemma 3. Define}

$$
\begin{aligned}
& \gamma_{k}(\xi)=\alpha\left(\epsilon^{-2 k} \xi_{1}+\epsilon^{-k} \xi_{2}+\epsilon^{-4 k} \xi_{3}\right)+\beta\left(\epsilon^{-5 k} \xi_{4}+\epsilon^{-6 k} \xi_{5}+\epsilon^{-3 k} \xi_{6}\right), \\
& \bar{\gamma}_{k}(\xi)=\beta\left(\epsilon^{2 k} \xi_{1}+\epsilon^{k} \xi_{2}+\epsilon^{4 k} \xi_{3}\right)+\alpha\left(\epsilon^{5 k} \xi_{4}+\epsilon^{6 k} \xi_{5}+\epsilon^{3 k} \xi_{6}\right),
\end{aligned}
$$


where $\alpha^{2}=(-1+\sqrt{ }-7) / \sqrt{ } 8=\left(\epsilon+\epsilon^{2}+\epsilon^{4}\right) / \sqrt{ } 2, \quad \beta=\bar{\alpha}, \quad \beta^{2}$ $=\left(\epsilon^{3}+\epsilon^{5}+\epsilon^{6}\right) / \sqrt{ } 2, \alpha \bar{\alpha}=\beta \bar{\beta}=1$, and $k=0, \cdots, 6$. One has (i) $R_{6}(G)$ induces on the $\gamma_{k}(\xi)$ and the $\bar{\gamma}_{k}$ respectively the degree seven permutation representation $R_{7}(G)$ of $G$ and its inverse $R_{7}^{-1}(G)$ and (ii)

$$
\begin{aligned}
\Sigma \gamma_{k}(\xi) & =\Sigma \bar{\gamma}_{k}(\xi)=0, \\
\gamma_{1-k}(\xi) & =(1 / \sqrt{ } 2)\left(\bar{\gamma}_{k}(\xi)+\bar{\gamma}_{k+1}(\xi)+\bar{\gamma}_{k+3}(\xi)\right), \\
\bar{\gamma}_{1-k}(\xi) & =(1 / \sqrt{ } 2)\left(\gamma_{k}(\xi)+\gamma_{k+1}(\xi)+\gamma_{k+3}(\xi)\right),
\end{aligned}
$$

where $k$ is computed $\bmod 7$, and

$$
\xi_{1}=(1 / 7 \alpha) \Sigma \epsilon^{2 k} \gamma_{k}(\xi), \cdots .
$$

Proof. (i) and (ii) follow from the substitution of (2) in the formulae in [1, p. 519] and [1, II, p. 459 and p. 501] and the corresponding facts noted there. However, once aware of (5), (6), (7) one easily verifies them directly. As for (i) one sees immediately that $T_{2}$ generates the cyclic permutation (6543210) of the indices of the $\gamma_{k}(\xi)$ and the inverse permutation of the $\bar{\gamma}_{k}(\xi)$. Calculation reveals that $U_{2}$ induces the (self-inverse) permutation (12)(36) on both $\gamma_{k}(\xi)$ and $\bar{\gamma}_{k}(\xi)$. These permutations generate the representations of (i). (7) implies their faithfulness.

\section{Invariants of $R_{6}(G)$ and main theorem.}

TheOREM A (GoRdAN [1]). Let $x_{k}, \bar{x}_{k}, k=0, \cdots, 6$ be two sets of variables which satisfy conditions (i) and (ii) of Lemma 3 when formally substituted for $\gamma_{k}(\xi), \bar{\gamma}_{k}(\xi)$ respectively. Then any (nonconstant, homogeneous) polynomial in $x_{0}, \cdots, x_{6}$ over the ring of integers which is invariant under $R_{7}(G)$ is a polynomial over $R(\sqrt{ } 2)$ ( $R$ is the rationals) in the power sums $S_{i}, \bar{S}_{i}, i=1, \cdots, 7$ of the $x$ 's and $\bar{x}^{\prime}$ ' (separately). By assumption $S_{1}=\bar{S}_{1}=0$. One easily verifies $S_{2}=\bar{S}_{2}$. The basis of eleven invariants $S_{2}, S_{j}, \bar{S}_{j}, j=3, \cdots, 7$ is irreducible, i.e., none is a polynomial (over $C$ ) in the others.

Gordan also computes explicitly a set of five syzygies but does not prove they are a basis for all syzygies.

Define, for a set of variables $\lambda_{1}, \cdots, \lambda_{6}$ and positive integral $j$

$$
S_{j}(\lambda)=\Sigma\left(\gamma_{k}(\lambda)\right)^{j}, \quad \bar{S}_{j}=\Sigma\left(\bar{\gamma}_{k}(\lambda)\right)^{j} .
$$

TheOREM 1. Let $t \in T^{3}$ (Teichmueller space of genus three) lie over $[S] \in M^{3}$ (modulus space of genus three), where $S$ is Klein's surface. One can introduce coordinates in $T^{3}$ near $t$ such that the local ring of $M^{3}$ at $[S]$ is generated by the irreducible basis of eleven distinct homogeneous polynomials obtained by setting $j=2, \cdots, 7$ in (8). 
Proof. Introducing $\lambda_{1}, \cdots, \lambda_{6}$ by Prescription III, using (2) as a basis for $A(S)$ ([6, p. 17-in Proposition 8 the reference should be to Prescription I, not II]), I have to find an irreducible basis for the nonconstant homogeneous polynomials in the $\lambda$ 's invariant under the hermitian adjoints of the matrices of $R_{6}(G)$. But by Lemma 2 and the group property this will be identical with a basis for the invariants of $R_{6}(G)$. Lemma 3 and Theorem A with

$$
x_{k}=\gamma_{k}(\lambda), \quad \bar{x}_{k}=\bar{\gamma}_{k}(\lambda), \quad k=0, \cdots, 6
$$

show that the invariants of Theorem 1 are a basis. The sticky point is that under the specialization (9) they might reduce. However, Gordan in [1, II, p. 461], says that even under the more severe specialization (9) and $\lambda_{1} \lambda_{2}=2 \lambda_{4}^{2}$, etc., the worst that can happen is $\beta S_{3}(\lambda)=\alpha \bar{S}_{3}(\lambda)$. A computation shows that this does not happen without the additional specialization.

\section{BIBLIOGRAPHY}

1. P. Gordan, Ueber Gleichungen siebenten Grades mit einer Gruppe von 168 Substitutionen, Math. Ann. 20 (1882), 515-530; II, ibid. 25 (1885), 459-521.

2. A. Hurwitz, Über algebraische Gebilde mit eindeutigen Transformationen in sich, Mathematische Werke, Bd. I, Birkhäuser, Basel, 1932, pp. 392-430.

3. F. Klein, Ueber die Transformation siebenter Ordnung der elliptischen Funktionen, Math. Ann. 14 (1879), 428-471.

4. A. M. Macbeath, On a theorem of Hurwitz. Proc. Glasgow Math. Assoc. 5 (1961), 90-96.

5. - On a curve of genus 7, Proc. London Math. Soc. 15 (1965), 527-542.

6. H. E. Rauch, $A$ transcendental view of the space of algebraic Riemann surfaces, Bull. Amer. Math. Soc. 71 (1965), 1-39.

Belfer Graduate School of Science, Yeshiva University 